\title{
Correction to: Exploring the potential of the platelet membrane proteome as a source of peripheral biomarkers for Alzheimer's disease
}

Laura E. Donovan', Eric B. Dammer², Duc M. Duong ${ }^{3}$, John J. Hanfelt ${ }^{4}$, Allan I. Levey ${ }^{1}$, Nicholas T. Seyfried ${ }^{1,3^{*}}$ and James J. Lah ${ }^{1 *}$

Correction to: Alz Res Therapy 5, 32 (2013)

https://doi.org/10.1186/alzrt186

It has recently come to our attention that a paper we published in 2013 in Alzheimer's Research \& Therapy never included the tables (Tables 1, 2 and 3) and supplemental tables (Additional file 1) in the published manuscript [1]. This must have been an oversight at the proof or production stage.

Tables 1, 2 and 3 are shown below.

\section{Supplementary Information}

The online version contains supplementary material available at https://doi. org/10.1186/s13195-021-00839-y.

Additional file 1: Supplementary Table S1. 1009 proteins identified

by unique peptides (top group members) identified in platelet

membrane proteome from either a probable AD pool or a cognitive

normal pool. Supplementary Table S2. 1957 total proteins (all potential group members) identified in platelet membrane proteome from either a probable AD pool or a cognitive normal pool. Supplementary Table S3. 144 potential marker proteins identified in platelet membrane proteome from a probable AD pool (FDR $<7 \%)$. Supplementary Table 4. 10 Classes incorporating 97 potentially novel AD biomarkers quantified in platelet membrane pools.

\section{Author details}

'Department of Neurology and Center for Neurodegenerative Disease,

Emory University School of Medicine, 615 Michael Street NE, Atlanta, GA

The original article can be found online at https://doi.org/10.1186/alzrt186.

*Correspondence: nseyfri@emory.edu; jlah@emory.edu

'Department of Neurology and Center for Neurodegenerative Disease,

Emory University School of Medicine, 615 Michael Street NE, Atlanta, GA

30322, USA

Full list of author information is available at the end of the article
30322, USA. ${ }^{2}$ Department of Human Genetics, Emory University School ofMedicine, 615 Michael Street NE, Atlanta, GA 30322, USA. ${ }^{3}$ Departmentof Biochemistry, Emory University School of Medicine, 1510 Clifton Road NE, Atlanta, GA 30322, USA. ${ }^{4}$ Department of Biostatistics and Bioinformatics, Emory University School of Medicine, 1518 Clifton Road NE, Atlanta, GA 30322, USA.

Published online: 18 May 2021

\section{Reference}

1. Donovan $L E$, Dammer EB, Duong DM, et al. Exploring the potential of the platelet membrane proteome as a source of peripheral biomarkers for Alzheimer's disease. Alz Res Therapy. 2013;5:32. https://doi.org/10.1186/alzrt1 86.

(c) The Author(s). 2021 Open Access This article is licensed under a Creative Commons Attribution 4.0 International License, which permits use, sharing, adaptation, distribution and reproduction in any medium or format, as long as you give appropriate credit to the original author(s) and the source, provide a link to the Creative Commons licence, and indicate if changes were made. The images or other third party material in this article are included in the article's Creative Commons licence, unless indicated otherwise in a credit line to the material. If material is not included in the article's Creative Commons licence and your intended use is not permitted by statutory regulation or exceeds the permitted use, you will need to obtain permission directly from the copyright holder. To view a copy of this licence, visit http://creativecommons.org/licenses/by/4.0/. The Creative Commons Public Domain Dedication waiver (http://creativecommons.org/publicdomain/zero/1.0/) applies to the data made available in this article, unless otherwise stated in a credit line to the data. 
Table 1 Participants

\begin{tabular}{lllllll}
\hline Controls & & & & \multicolumn{3}{l}{ Probable Alzheimer's Disease (AD) } \\
\cline { 1 - 3 } \cline { 5 - 6 } Case \# & Age & MMSE $^{\mathrm{a}}$ & & Case \# & Age & MMSE $^{\mathrm{a}}$ \\
\hline 1 & 75 & 28 & $\mathbf{1}$ & $\mathbf{8 3}$ & $\mathbf{2 0}$ \\
$\mathbf{2}$ & $\mathbf{7 2}$ & $\mathbf{2 9}$ & $\mathbf{2}$ & $\mathbf{7 7}$ & $\mathbf{1 8}$ \\
$\mathbf{3}$ & $\mathbf{7 4}$ & $\mathbf{3 0}$ & $\mathbf{3}$ & $\mathbf{9 0}$ & $\mathbf{1 1}$ \\
$\mathbf{4}$ & $\mathbf{6 7}$ & $\mathbf{2 9}$ & $\mathbf{4}$ & $\mathbf{8 3}$ & $\mathbf{2 0}$ \\
$\mathbf{5}$ & $\mathbf{7 3}$ & $\mathbf{2 9}$ & $\mathbf{5}$ & $\mathbf{8 2}$ & $\mathbf{2 4}$ \\
$\mathbf{6}$ & $\mathbf{6 0}$ & $\mathbf{3 0}$ & $\mathbf{6}$ & 61 & 12 \\
7 & 69 & 30 & 7 & 55 & 25 \\
AVERAGES: & 70.00 & 29.3 & & 75.86 & 18.6 \\
\hline
\end{tabular}

Cases in bold were pooled for proteomic analysis

Bold type-face indicates cases pooled for proteomic analysis

${ }^{a}$ MMSE mini mental status exam, clinical measurement of cognitive function
Table 3 Overlap of AD platelet membrane protein changes with previously proposed mechanistic and diagnostic biomarkers

\begin{tabular}{llll}
\hline $\begin{array}{l}\text { Mean } \log _{2} \\
\text { (AD/CT) }\end{array}$ & $\begin{array}{l}\text { R1 vs R2 } \\
\text { CV, SD\% }\end{array}$ & Symbol & Protein Name \\
\hline 2.46 & $14 \%$ & MGAT4B & $\begin{array}{l}\text { mannosyl (alpha-1,3-)-glycoprotein } \\
\text { beta-1,4-N-acetylglucosaminyltransferase, } \\
\text { isozyme B }\end{array}$ \\
-1.23 & $52 \%$ & VPS13C & $\begin{array}{l}\text { vacuolar protein sorting 13 homolog C } \\
\text { (S. cerevisiae) }\end{array}$ \\
-1.24 & $20 \%$ & AGPS & $\begin{array}{l}\text { alkylglycerone phosphate synthase } \\
\text { ferritin, light polypeptide }\end{array}$ \\
-1.92 & $15 \%$ & FTL & insulin-like growth factor 1 receptor \\
\hline
\end{tabular}

Table 2 DAVID analysis of changes in proteins associated with platelet-specific secretion

\begin{tabular}{|c|c|c|c|c|}
\hline $\begin{array}{l}\text { Mean } \log _{2} \\
(A D / C T)\end{array}$ & $\begin{array}{l}\text { R1 vs R2 } \\
\text { CV, SD\% }\end{array}$ & Symbol & Protein Name & $\begin{array}{l}\text { Ontology } \\
\text { Groups }\end{array}$ \\
\hline-1.70 & $0.6 \%$ & FGA & fibrinogen alpha chain & $A, B, C, E, F$ \\
\hline-2.03 & $7.5 \%$ & $\mathrm{FGB}$ & fibrinogen beta chain & $A, B, C, E, F$ \\
\hline-1.69 & $1.5 \%$ & FGG & fibrinogen gamma chain & $A, B, C, E, F$ \\
\hline-2.02 & $1.9 \%$ & WWF & von Willebrand factor & $A, B, C, E$ \\
\hline-1.23 & $39 \%$ & PROS1 & protein S (alpha) & $B, C, E, F$ \\
\hline-1.19 & $59 \%$ & FN1 & fibronectin 1 & $B, C, D, F$ \\
\hline-1.19 & $41 \%$ & SERPINA1 & $\begin{array}{l}\text { serpin peptidase inhibitor, } \\
\text { clade A (alpha-1 antiproteinase, } \\
\text { antitrypsin), member } 1\end{array}$ & $B, C, E$ \\
\hline-1.47 & $31 \%$ & SPARC & $\begin{array}{l}\text { secreted protein, acidic, } \\
\text { cysteine-rich (osteonectin) }\end{array}$ & $B, C, F$ \\
\hline-2.05 & $0.9 \%$ & THBS1 & thrombospondin 1 & $B, C, F$ \\
\hline 1.47 & $1.2 \%$ & GP9 & glycoprotein IX (platelet) & $B, C$ \\
\hline-2.82 & $2.9 \%$ & C4BPA & $\begin{array}{l}\text { complement component } 4 \\
\text { binding protein, alpha }\end{array}$ & $D, E$ \\
\hline-1.24 & $94 \%$ & SERPINE1 & $\begin{array}{l}\text { serpin peptidase inhibitor, } \\
\text { clade } \mathrm{E} \text { (nexin, plasminogen } \\
\text { activator inhibitor type 1), } \\
\text { member } 1\end{array}$ & $E, F$ \\
\hline-1.83 & $66 \%$ & TF & transferrin & C \\
\hline-1.65 & $10 \%$ & $\mathrm{HP}$ & $\begin{array}{l}\text { haptoglobin-related protein; } \\
\text { haptoglobin }\end{array}$ & D \\
\hline-1.33 & $31 \%$ & SUSD1 & sushi domain containing 1 & $\mathrm{D}$ \\
\hline
\end{tabular}

